\title{
THE DETROIT MEETING OF THE AMERICAN ASSOCIATION FOR THE ADVANCEMENT OF SCIENCE.
}

The forty-sixth annual meeting of the American Association for the Advancement of Science was held at Detroit, August 9-16. There were 290 members and associates registered, including over twenty foreign scientists.

The officers of Section A were as follows: Vice-President, W. W. Beman; Secretary, J. McMahon; Press Secretary, P. A. Lambert; Councillor, E. W. Hyde; Sectional Committee, W. W. Beman, J. McMahon, A. Macfarlane, W. F. Durand, J. E. Kershner, W. S. Pritchett; Member of Nominating Committee, A. Ziwet; Committee to Nominate Officers of Section, W. W. Beman, J. McMahon, A. Hall, Jr., R. S. Woodward, A. Macfarlane.

The Vice-President delivered an address "On a chapter in the history of mathematics."

The following papers were presented :-

(1) "A problem in substitution-groups." By Dr. G. A. Miller, Ann Arbor, Mich.

(2) "Continuous groups of spherical transformations in space." By Professor H. B. Newson, Lawrence, Kans.

(3) "Treatment of differential equations by approximate methods." By Professor W. F. Durand, Ithaca, N. Y.

(4) "Commutative matrices." By Professor J. B. SHAw, Jacksonville, Ill.

(5) "On the theory of the quadratic equation." By Professor A. Macfarlane, Lehigh University, South Bethlehem, Pa.

(6) "A new principle in solving certain linear differential equations that occur in mathematical physics." By Professor A. Macfarlank, Lehigh University, South Bethlehem, Pa.

(7) "Condition that the line common to $n-1$ planes in an $n$-space may pierce a given quadric surface in the same space." By Dr. VIrgil SNyder, Ithaca, N. Y.

(8) "The psychology of the personal equation." By Professor T. H. SAFFoRD, Williamstown, Mass.

(9) "Compound determinants." (Preliminary communication.) By Professor W. H. Metzler, Syracuse, N. Y. (10) "Waters within the earth and laws of rainflow." By W. S. Auchincloss, C.E. Philadelphia, Pa.

(11) "On the secular motion of the earth's magnetic 
axis." By Dr. L. A. Bauer, University of Cincinnati, Cincinnati, $\mathrm{O}$.

(12) "Simple expressions for the diurnal range of the magnetic declination and of the magnetic inclination." By Dr. L. A. Bauer, University of Cincinnati, Cincinnati, $O$.

(13) "The theory of perturbations and Lie's theory of contact-transformations." By Dr. E, O. Lovetr, Princeton, N. J.

(14) "On rational right triangles." No. 1. By Dr. Artemas Martin, U. S. Coast Survey, Washington, D. C.

(15) "Some results in integration expressed by the elliptic integrals." By Professor James McMahon, Cornell University, Ithaca, N. Y.

(16) "Modification of the Eulerian cycle due to inequality of the equatorial moments of inertia of the earth." By Professor R. S. Woodward, Columbia University, N. Y. (17) "Integration of the equations of rotation of a nonrigid mass for the case of equal principal moments of inertia." By Professor R. S. WoodwARd, Columbia University, N. Y.

(18) "General theorems concerning a certain class of functions deduced from the properties of the Newtonian potential function." By Dr. J. W. Glover, Ann Arbor, Mich.

(19) "The importance of adopting standard systems of notation and coördinates in mathematics and physics." By Professor Frank H. Bigelow, U. S. Weather Bureau, Washington, D. C.

(20) "A remarkable complete quadrilateral among the Pascal lines of an inscribed six-point of a conic." By Professor R. D. Bohannan, Columbus, $O$.

(21) "Stereoscopic views of spherical catenaries and gyroscopic curves." By Professor A. G. Greenhill, Royal Artillery College, Woolwich.

In No. 1 it was pointed out that to every simple isomorphism of a group to itself corresponds some substitution of its operators, and that to all such isomorphisms corresponds a substitution group, called the group of isomorphisms of the given group. A new and simple proof was given of the following theorem of Jordan's: When a regular group $(R)$ of order $n$ is transformed into itself by the largest possible group $(L)$ of its own degree, the subgroup of $L$, which includes all its substitutions that do not contain a given element, is the group of isomorphisms of $R$.

Isomorphisms which may be derived from a given one by means of real transforming operators are said to be trans- 
form to each other. All simple isomorphisms of $R$ to itself are transforms of the identical isomorphism. Isomorphisms that do not belong to the same system of transforms are said to be distinct. To the transforms of the identical isomorphisms corresponds a subgroup of the group of isomorphisms. The number of substitutions of this group that correspond to an isomorphism which is distinct from the identical is a multiple of the order of this subgroup.

In No. 2 the general group is that of Lie's Kugelgeometrie. All infinity in space is regarded as a single point as in the complex plane, and all planes are regarded as spheres through the point at infinity. The general group is ten-fold. All transformations leaving a point invariant form a sevenfold subgroup. There is a sixfold subgroup leaving a sphere invariant. This is identical with the sixfold group of circular transformations on the Neumann sphere or in the complex plane.

No. 3 showed how successive values of a function defined by a differential equation could be numerically computed without solving the equation. Using Newton's notation for derivatives and letting subscripts correspond to successive points (or values of $x$ ), the simple trapezoidal rule expresses approximately the difference between $\dot{y}_{0}$ and $\dot{y}_{1}$ in terms of $\ddot{y}_{0}$ and $\ddot{y}_{1}$, and similarly the difference between $y_{0}$ and $y_{1}$ in terms of $\dot{y}_{0}$ and $\dot{y}_{1}$. Thus $\dot{y}_{1}$ and $y_{1}$ may be ultimately expressed in terms of $y_{0}, \dot{y}_{0}, \ddot{y}_{0}, . \ddot{y}_{1}$; and then the differential equation may be solved for $\ddot{y}_{1}$ in terms of $y_{0}, \dot{y}_{0}, \ddot{y}_{0}$. This value substituted back will give the values of $y_{1}, y_{1}$. Thus the circumstances for the point (1) are known when those for the initial point are assigned; and so on from point to point. The trapezoidal rule may be replaced by more accurate ones such as Simpson's rule. The method applies to equations of any order and degree, and to simultaneous equations.

No. 4 showed that if two matrices are commutative, i.e., $\varphi \psi=\psi \varphi$, then there is no latent region of any root of the one which lies in the extension composed of two or more latent regions of a root of the other unless it includes the entirety of these regions. The character of the conditions arising from the forms of the partial matrices governing the latent regions when one of the matrices has equal roots was developed.

No. 5 stated the current theory of the equation $x^{2}+2 b x$ $+c=0$, as follows: When $b^{2}>c$, the radical term in the value of $x$ is to be simply added to the other term, and is 
represented as part of the same straight line; but when $b^{2}<c$, the radical is to be geometrically added to the other term, and is represented at right angles to it. The author extended this theory as follows: When $b^{2}>c$ the radical may be represented at right angles, so that the two terms of the root represent a hyperbolic complex quantity. This interpretation is supplementary to the idea of a circular complex quantity. In both cases the root is complex. Again when $b^{2}<c, x$ may not be complex, but may be scalar, in which case the two parts of the root are represented along the same straight line. The author illustrated this by the cosine of an angle which is the sum of a circular and a complex angle.

In No. 6 the author used, as an example, the equation

$$
L \frac{d i}{d t}+R i=E \sin \omega t
$$

and showed that when, as here, the right-hand member is the orthogonal projection of a plane motion, it is easier to pass to the auxiliary motion using planar algebra than it is to proceed with the given equation directly.

In No. 7 the analytical problem was to determine the reality of the values which simultaneously satisfy $n-1$ linear equations in $n$ variables, and a quadratic equation in the same $n$ variables. The reality of the roots depends upon the sign of a symmetrical determinant of order $n-1$, whose elements are quadratic functions of the coefficients of the given equations; and the criterion applies, whether $n$ is odd or even. The theorem has important applications when $n$ equals $2,3,4$ or 5 and may be employed indifferently, whether the point, the plane, the line, the circle or the sphere be taken as the generating element.

The object of No. 8 was to awaken an interest among astronomers and psychologists such as to induce them to pay more attention to each other's work and to improve their own methods.

In No. 9 the idea of obtaining the value of $A(m)$ the $m$ th compound of the determinant $A$ as a power of $A$, by multiplying it by its adjugate $A(n-m)$, the $(n-m)$ th compound of $A$, is extended to finding the value of certain minors of $A(m)$ in ternos of $A$ and its minors. Taking any minor of $A$ and finding its $m$ th and its $(n-m)$ th compounds, their values in terms of the minor is in the same way found. Making use of this principle and the laws of Complementaries and Extensible Minors it is shown how the value of many minors of $A(m)$ may be found, and by making use of a com- 
prehensive notation the whole subject is unified, the laws of vanishing minors set forth and such well known theorems as Sylvester's and others are easily established.

No. 10 was read by title and a printed pamphlet distributed.

No. 11 stated that 70 per cent. of the total magnetization of the earth can be referred to a homogeneous magnetization about a diameter inclined to the earth's rotation axis at an angle of about $12^{\circ}$. This axis has been termed by Gauss the earth's magnetic axis. It is an interesting question to determine the motion of the axis during the past two or three centuries; and the author attempted to solve this problem as far as is possible with the data at present at command.

In No. 12 the author stated that no formulæ had until now been found by which the diurnal range of the magnetic declination, for example, could be computed for various portions of the earth. He had recently found the following most simple formulæ to hold true within the fluctuations to which the quantities, themselves are subject:

$$
\begin{aligned}
& \beta_{d}=2^{\prime} .58 \sec ^{2} \varphi \\
& \beta_{i}=\frac{6^{\prime} .1}{1+3 \sin ^{2} \varphi}
\end{aligned}
$$

where $\beta_{d}=$ diurnal range of declination,

$\beta_{i}=$ diurnal range of inclination,

$\varphi=$ magnetic latitude found from the equation

$$
\tan \varphi=\frac{1}{2} \tan I,
$$

$I=$ magnetic dip.

Equation (1) was found in the first place empirically, then under certain assumptions deduced theoretically. Equation (2) was then deduced theoretically and found to satisfy the data.

No. 13 followed the theory of perturbations in the problems of mechanics in the order of its historical development from Lagrange to Lie with a view to the final presentation of the theory in its just position as one phase of Lie's theory of contact-transformations.

No. 14 gave a brief bibliography of the Pythagorean proposition, and an algebraic solution in general terms of the equation $x^{2}+y^{2}=z^{2}$. It closed with an extensive numerical table of rational right-triangles.

No. 15 expressed in terms of the three kinds of elliptic integrals some algebraic integrals which had, apparently, never been completely worked out. 
No. 16 showed how to express the effect of a small difference in the equatorial moments of inertia of the earth on the period of revolution of the instantaneous axis of rotation around the axis of figure. A remarkable value was obtained for the average angular velocity of that revolution; and a formula was deduced for the difference in the equatorial moments essential to explain the discrepancy between the observed and computed values of the Eulerian cycle.

In No. 17 the case considered was that of no applied forces, or that in which there is conservation of moment of momentum. The problem is of practical interest in its application to the question of variation of latitudes on the earth. Several new theorems with respect to the motions of the mass were derived.

No. 18 derived various properties of a class of functions which include the spherical harmonics as a particular case.

No. 19 described the present annoying state of the subject matter of notation and coördinate systems, especially in Mathematical Physics, and advocated the adoption of certain standards and fundamental conventions. The matter will be considered again at the Boston meeting, when a large number of representative mathematicians will probably attend.

No. 20 pointed out a certain complete quadrilateral whose properties throw new light on the theory of the Pascal lines connected with an inscribed hexagon of a conic.

In No. 21 Professor Greenhill kindly consented to exhibit some stereoscopic views of certain interesting curves in space, and pointed out their bearing on some parts of the theory of elliptic functions. It may be mentioned here that the section is also indebted to Professor Greenhill for instructive remarks made in connection with many of the other papers.

In the absence of their authors Nos. 1, 7, 11, 12, 13 and 14 were presented in abstract by the Secretary, who also read No. 8 in full. No. 9 was briefly presented by Professor Shaw.

The semi-centennial meeting of the Association will be held in Boston, beginning August 22, 1898, under the presidency of F. W. Putnam. The officers elected for Section A are E. E. Barnard, of Yerkes Observatory, and Alexander Ziwet, of Ann Arbor.

James MoMahon.

Cornell University. 